Proyecciones Journal of Mathematics

Vol. 35, No 3, pp. 289-300, September 2016.

Universidad Católica del Norte

Antofagasta - Chile

\title{
Weak forms of continuity and openness
}

\author{
Miguel Caldas \\ Universidade Federal Fluminense, Brasil \\ and \\ Saeid Jafari \\ College of Vestsjaelland South, Denmark \\ Received : January 2016. Accepted : May 2016
}

\begin{abstract}
Some new class of functions, called somewhat $\delta$-precontinuous, somewhat $\delta$-preopen and hardly $\delta$-preopen functions, have been defined and studied by utilizing $\delta$-preopen sets. Moreover, characterizations and properties of these functions are presented.
\end{abstract}

2000 Mathematics Subject Classification : $54 A 40$.

Key words and phrases : Topological spaces, $\delta$-preopen sets, $\delta$ precontinuity, somewhat $\delta$-precontinuity. 


\section{Introduction and Preliminaries}

Recent progress in the study of characterizations and generalizations of continuity has been done by means of several generalized closed sets. The first step of generalizing closed set was done by Levine in 1970 [9]. The notion of generalized closed sets has been studied extensively in recent years by many topologists since generalized closed sets are the only natural generalization of closed sets. More importantly, they also suggest several new properties of topological spaces. As a generalization of closed sets, $\delta$ preclosed sets were introduced and studied by Raychaudhuri and Mukherjee ([10], [11], [12]). This notion was further studied by ([1], [2], [3], [4], [5], [6]). In this paper somewhat $\delta$-precontinuous, somewhat $\delta$-preopen and hardly $\delta$-preopen functions are introduced. Further, we obtain some results which are similar to those of somewhat continuous, somewhat open and hardly open functions.

Throughout the paper $(X, \tau)$ and $(Y, \sigma)$ (or simply $X$ and $Y$ ) represent topological spaces on which no separation axioms are assumed unless otherwise mentioned. For a subset $A$ of a space $(X, \tau), \operatorname{cl}(A), \operatorname{int}(A)$ and $X \backslash A$ denote the closure of $A$, the interior of $A$ and the complement of $A$ in $X$, respectively. A point $x \in X$ is called a $\delta$-cluster point of $A$ [13] if $\operatorname{int}(\operatorname{cl}(V)) \cap A \neq \emptyset$ for every open set $V$ of $X$ containing $x$. The set of all $\delta$-cluster points of $A$ is called the $\delta$-closure of $A$ and is denoted by $\delta c l(A)$ ). If $A=\delta c l(A)$ ), then $A$ is said to be $\delta$-closed. The complement of a $\delta$-closed set is said to be $\delta$-open [13]. The union of all $\delta$-open sets contained in a subset $A$ is called the $\delta$-interior of $A$ and is denoted by $\operatorname{\delta int}(A)$.

A subset $A$ of a topological space $X$ is said to be $\delta$-preopen [11] if $A \subset$ $\operatorname{int}(\delta c l(A))$. The complement of a $\delta$-preopen set is said to be $\delta$-preclosed. The intersection (union) of all $\delta$-preclosed ( $\delta$-preopen) sets containing (contained in) $A$ in $X$ is called the $\delta$-preclosure $(\delta$-preinterior) of $A$ and is denoted by $\delta p c l(A)$ (resp. $\delta p i n t(A))$. By $\delta P O(X)$ or $\delta P O(X, \tau)$ (resp. $\delta P C(X))$, we denote the collection of all $\delta$-preopen (resp. $\delta$-preclosed) sets of $X$. We set $\delta P O(X, x)=\{U: x \in U \in \delta P O(X)\}$.

Lemma 1.1. [11]. The following properties holds for the $\delta$-preclosure of a set in a space $X$ :

(1) Arbitrary union (intersection) of $\delta$-preopen ( $\delta$-preclosed) sets in $X$ is $\delta$-preopen (resp. $\delta$-preclosed).

(2) $A$ is $\delta$-preclosed in $X$ if and only if $A=\delta p c l(A)$.

(3) $\delta p c l(A) \subset \delta p c l(B)$ whenever $A \subset B(\subset X)$.

(4) $\delta p c l(A)$ is $\delta$-preclosed in $X$. 
(5) $\delta p c l(\delta p c l(A))=\delta p c l(A)$.

(6) $\delta p c l(A)=\{x \in X \mid U \cap A \neq \emptyset$ for every $\delta$-preopen set $U$ containing $x\}$.

(7) $\operatorname{\delta pcl}(A)=A \cup \operatorname{cl}(\operatorname{\delta int}(A))$.

(8) If $A$ is $\delta$-open, then $\delta p c l(A)=\operatorname{cl}(A)$.

(9) $\delta p c l(X \backslash A)=X \backslash \delta \operatorname{pint}(A)$.

Recall that, a subset $A$ of a space $(X, \tau)$ is called preopen (resp. regular open) if $A \subset \operatorname{int}(\operatorname{cl}(A))$ (resp. $A=\operatorname{int}(\operatorname{cl}(A))$ ).

We have the following diagram in which the converses of implications need not be true.

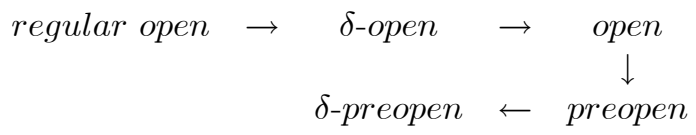

Definition 1. A space $(X, \tau)$ is a $\delta$-door space if every subset of $X$ is $\delta$-open or $\delta$-closed.

Theorem 1.2. If a space $(X, \tau)$ is a $\delta$-door space, then every $\delta$-preopen set in $(X, \tau)$ is $\delta$-open.

Proof. Asume that $A$ is a $\delta$-preopen set in $(X, \tau)$. Now if $A$ is not $\delta$ open, then it is $\delta$-closed. Then $A=\delta c l(A)$. Therefore $\operatorname{int}(A)=\operatorname{int}(\delta c l(A))$. This means that $A \not \subset \operatorname{int}(\delta c l(A))$. But this is a contradiction. Hence the claim.

It should be noted that if a topological space $(X, \tau)$ is a $\delta$-door space, then each singleton in $\mathrm{X}$ is either $\delta$-open or $\delta$-closed. A subset $E$ of a topological space $(X, \tau)$ is said to be $\delta$-predense in $X$ if there is no proper $\delta$-preclosed set $C$ in $X$ such that $E \subset C \subset X$.

Theorem 1.3. For a space $(X, \tau)$, the following are equivalent:

(1) Every $\delta$-preopen set is $\delta$-open;

(2) Every $\delta$-predense set is $\delta$-open.

\section{Somewhat $\delta$-precontinuous functions}

Definition 2. A function $f:(X, \tau) \rightarrow(Y, \sigma)$ is said to be somewhat $\delta$ precontinuous if for each $V \in \sigma$ with nonempty inverse, there exists a nonempty set $U \in \delta P O(X, \tau)$ such that $U \subset f^{-1}(V)$. 
Definition 3. A function $f:(X, \tau) \rightarrow(Y, \sigma)$ is called somewhat continuous [7], if for each $V \in \sigma$ with nonempty inverse, there exists a nonempty open set $U$ of $X$ such that $U \subset f^{-1}(V)$.

It is clear that every continuous function is somewhat continuous and every somewhat continuous is somewhat $\delta$-precontinuous. But the converses are not true as it is shown in the following examples.

Example 2.1. ([7], Example 1) Let $X=\{a, b, c\}$ with topologies $\tau=$ $\{\emptyset,\{b\},\{a, c\}, X\}$ and $\sigma=\{\emptyset,\{a, b\}, X\}$. Then the identity function $f$ : $(X, \tau) \rightarrow(Y, \sigma)$ is somewhat continuous but it is not continuous.

Example 2.2. Let $X=\{a, b, c\}$ with topologies $\tau=\{\emptyset,\{a\},\{b, c\}, X\}$ and $\sigma=\{\emptyset,\{a\}, X\}$. Define a function $f:(X, \tau) \rightarrow(X, \sigma)$ by $f(a)=c$, $f(b)=a$ and $f(c)=b$. Then $f$ is somewhat $\delta$-precontinuous. But $f$ is not somewhat continuous.

Theorem 2.3. For a surjective function $f:(X, \tau) \rightarrow(Y, \sigma)$, the following statements are equivalent:

(i) $f$ is somewhat $\delta$-precontinuous;

(ii) If $C$ is a closed subset of $Y$ such that $f^{-1}(C) \neq X$, then there is a proper $\delta$-preclosed subset $F$ of $X$ such that $f^{-1}(C) \subset F$;

(iii) If $E$ is a $\delta$-predense subset of $X$, then $f(E)$ is a dense subset of $Y$.

Proof. $(i) \Rightarrow(i i)$ : Let $C$ be a closed subset of $Y$ such that $f^{-1}(C) \neq X$. Then $Y \backslash C$ is an open set in $Y$ such that $f^{-1}(Y \backslash C)=X \backslash f^{-1}(C) \neq \emptyset$. By (i), there exists a nonempty $\delta$-preopen set $U$ in $X$ such that $U \subset$ $f^{-1}(Y \backslash C)=X \backslash f^{-1}(C)$. This means that $f^{-1}(C) \subset X \backslash U$ and $X \backslash U=F$ is a proper $\delta$-preclosed set in $X$.

$($ ii $) \Rightarrow(i)$ : Let $V \in \sigma$ and $f^{-1}(V) \neq \emptyset$. Then $Y \backslash V$ is closed and $f^{-1}(Y \backslash V)=X \backslash f^{-1}(V) \neq X$. By (ii), there exists a proper $\delta$-preclosed set $F$ of $X$ such that $f^{-1}(Y \backslash V) \subset F$. This implies that $X \backslash F \subset f^{-1}(V)$ and $X \backslash F \in \delta P O(X)$ with $X \backslash F \neq \emptyset$.

$($ ii $) \Rightarrow($ iii $)$ : Let $E$ be a $\delta$-predense set in $X$. Suppose that $f(E)$ is not dense in $Y$. Then there exists a proper closed set $C$ in $Y$ such that $f(E) \subset C \subset Y$. Clearly $f^{-1}(C) \neq X$. By (ii), there exists a proper 
$\delta$-preclosed subset $F$ such that $E \subset f^{-1}(C) \subset F \subset X$. This is a contradiction to the fact that $E$ is $\delta$-predense in $X$.

$($ iii $) \Rightarrow($ ii): Suppose (ii) is not true. This means that there exists a closed set $C$ in $Y$ such that $f^{-1}(C) \neq X$ and there is no proper $\delta$ preclosed set $F$ in $X$ such that $f^{-1}(C) \subset F$. This means that $f^{-1}(C)$ is $\delta$-predense in $X$. But by (iii), $f\left(f^{-1}(C)\right)=C$ must be dense in $Y$, which is a contradiction to the choice of $C$.

Definition 4. If $X$ is a set and $\tau$ and $\tau^{*}$ are topologies on $X$, then $\tau$ is said to be $\delta$-preequivalent (resp. equivalent [7]) to $\tau^{*}$ provided if $U \in \tau$ and $U \neq \emptyset$, then there is a $\delta$-preopen (resp. open) set $V$ in $\left(X, \tau^{*}\right)$ such that $V \neq \emptyset$ and $V \subset U$ and if $U \in \tau^{*}$ and $U \neq \emptyset$ then there is a $\delta$-preopen (resp. open) set $V$ in $(X, \tau)$ such that $V \neq \emptyset$ and $V \subset U$

Now consider the identity function $f:(X, \tau) \rightarrow\left(X, \tau^{*}\right)$ and assume that $\tau$ and $\tau^{*}$ are $\delta$-preequivalent. Then $f:(X, \tau) \rightarrow\left(X, \tau^{*}\right)$ and $f^{-1}$ : $\left(X, \tau^{*}\right) \rightarrow(X, \tau)$ are somewhat $\delta$-precontinuous. Conversely, if the identity function $f:(X, \tau) \rightarrow\left(X, \tau^{*}\right)$ is somewhat $\delta$-precontinuous in both directions, then $\tau$ and $\tau^{*}$ are $\delta$-preequivalent.

Theorem 2.4. If $f:(X, \tau) \rightarrow(Y, \sigma)$ is a surjection somewhat $\delta$-precontinuous and $\tau^{*}$ is a topology for $X$, which is $\delta$-preequivalent to $\tau$, then $f:\left(X, \tau^{*}\right) \rightarrow$ $(Y, \sigma)$ is somewhat $\delta$-precontinuous.

Proof. Let $V$ be an open subset of $Y$ such that $f^{-1}(V) \neq \emptyset$. Since $f:(X, \tau) \rightarrow(Y, \sigma)$ is somewhat $\delta$-precontinuous, there exists a nonempty $\delta$-preopen set $U$ in $(X, \tau)$ such that $U \subset f^{-1}(V)$. But by hypothesis $\tau^{*}$ is $\delta$-preequivalent to $\tau$. Therefore, there exists a $\delta$-preopen set $U^{*}$ in $\left(X, \tau^{*}\right)$ such that $U^{*} \subset U$. But $U \subset f^{-1}(V)$. Then $U^{*} \subset f^{-1}(V)$; hence $f$ : $\left(X, \tau^{*}\right) \rightarrow(Y, \sigma)$ is somewhat $\delta$-precontinuous.

Theorem 2.5. Let $f:(X, \tau) \rightarrow(Y, \sigma)$ be a somewhat $\delta$-precontinuous surjection and $\sigma^{*}$ be a topology for $Y$, which is equivalent to $\sigma$. Then $f:(X, \tau) \rightarrow\left(Y, \sigma^{*}\right)$ is somewhat $\delta$-precontinuous.

Proof. Let $V^{*}$ be an open subset of $\left(Y, \sigma^{*}\right)$ such that $f^{-1}\left(V^{*}\right) \neq \emptyset$. Since $\sigma^{*}$ is equivalent to $\sigma$, there exists a nonempty open set $V$ in $(Y, \sigma)$ such that $V \subset V^{*}$. Now $\emptyset \neq f^{-1}(V) \subset f^{-1}\left(V^{*}\right)$. Since $f:(X, \tau) \rightarrow(Y, \sigma)$ is somewhat $\delta$-precontinuous, there exists a nonempty $\delta$-preopen set $U$ in $(X, \tau)$ such that $U \subset f^{-1}(V)$. Then $U \subset f^{-1}\left(V^{*}\right)$; hence $f:(X, \tau) \rightarrow$ $\left(Y, \sigma^{*}\right)$ is somewhat $\delta$-precontinuous. 
Theorem 2.6. Let $f:(X, \tau) \rightarrow(X, \sigma)$ be a function and $X=A \cup B$, where $A, B \in \tau$. If the restriction function $f_{A}:\left(X, \tau_{A}\right) \rightarrow(X, \sigma)$ and $f_{B}:\left(X, \tau_{B}\right) \rightarrow(X, \sigma)$ are somewhat $\delta$-precontinuous, then $f$ is somewhat $\delta$-precontinuous.

Proof. Let $U$ be any open subset of $Y$ such that $f^{-1}(U) \neq \emptyset$. then $\left(f_{A}\right)^{-1}(U) \neq \emptyset$ or $\left(f_{B}\right)^{-1}(U) \neq \emptyset$ or both $\left(f_{A}\right)^{-1}(U) \neq \emptyset$ and $\left(f_{B}\right)^{-1}(U) \neq$ $\emptyset$. Suppose $\left(f_{A}\right)^{-1}(U) \neq \emptyset$. Since $f_{A}:\left(X, \tau_{A}\right) \rightarrow(X, \sigma)$ is somewhat $\delta$ precontinuous, there exists a $\delta$-preopen set $V$ in $A$ such that $V \neq \emptyset$ and $V \subset\left(f_{A}\right)^{-1}(U) \subset f^{-1}(U)$. Since $V$ is $\delta$-preopen in $A$ and $A$ is open in $X$, $V$ is $\delta$-preopen in $X$. Therefore $f$ is somewhat $\delta$-precontinuous.

The proof of the other cases are similar.

Theorem 2.7. If $f:(X, \tau) \rightarrow(X, \sigma)$ is somewhat $\delta$-precontinuous and $g:(X, \sigma) \rightarrow(X, \eta)$ is continuous, then $f \circ g:(X, \tau) \rightarrow(Z, \eta)$ is somewhat $\delta$-precontinuous.

\section{Two form of weak openness}

In this section we define and characterize two new weak forms of openness, i.e., the somewhat $\delta$-preopen and hardly $\delta$-preopen functions.

Definition 5. A function $f:(X, \tau) \rightarrow(Y, \sigma)$ is said to be somewhat $\delta$ preopen if for each nonempty set $U \in \tau$, then there exists a nonempty $\delta$-preopen set $V$ in $Y$ such that $V \subset f(U)$.

Example 3.1. Let $X=\{a, b, c\} \tau=\{\emptyset,\{a, b\}, X\}, Y=\{1,2,3\}$ and $\sigma=\{\emptyset,\{1,3\}, Y\}$. Define a function $f:(X, \tau) \rightarrow(Y, \sigma)$ by $f(a)=2$, $f(b)=3$ and $f(c)=1$. Then $f$ is somewhat $\delta$-preopen.

It is clear that every somewhat open function is somewhat $\delta$-preopen. But the converse is not true as it is shown by the following example.

Example 3.2. Let $X=\{a, b, c\} \tau=\{\emptyset,\{a, b\}, X\}, Y=\{1,2,3\}$ and $\sigma=\{\emptyset,\{2,3\}, Y\}$. Define a function $f:(X, \tau) \rightarrow(Y, \sigma)$ by $f(a)=1$, $f(b)=2$ and $f(c)=3$. Then $f$ is somewhat $\delta$-preopen. But $f$ is not somewhat open.

We have the following obvious characterization of somewhat $\delta$-preopenness.

Theorem 3.3. A function $f:(X, \tau) \rightarrow(Y, \sigma)$ is somewhat $\delta$-preopen if and only if for any $A \subset X$, $\operatorname{int}(A) \neq \emptyset$ implies that $\delta p i n t(f(A)) \neq \emptyset$. 
It should be noted that if we have a topological space $(X, \tau)$ in which $A \in \tau$ or $A$ has empty interior ( a rare set) for every $A \in X$, then $\tau=\tau^{\alpha}=$ $\tau^{s}$, where $\tau^{\alpha}$ and $\tau^{s}$ are the collection of $\alpha$-open sets and semi-open sets respectively.

Theorem 3.4. For a function $f:(X, \tau) \rightarrow(Y, \sigma)$, the following statements are equivalent:

(i) $f$ is somewhat $\delta$-preopen;

(ii) If $D$ is a $\delta$-predense subset of $Y$, then $f^{-1}(D)$ is a dense subset of $X$.

Proof. $(i) \Rightarrow(i i)$ : Suppose $D$ is a $\delta$-predense set in $Y$. We want to show that $f^{-1}(D)$ is a dense subset of $X$. Suppose that $f^{-1}(D)$ is not dense in $X$. Then there exists a closed set $B$ in $X$ such that $f^{-1}(D) \subset B \subset X$. By (i), since $X \backslash B$ is open, there exists a nonempty $\delta$-preopen subset $E$ in $Y$ such that $E \subset f(X \backslash B)$. Therefore $E \subset f(X \backslash B) \subset f\left(f^{-1}(Y \backslash D)\right) \subset Y \backslash D$. That is, $D \subset Y \backslash E \subset Y$. Now, $Y \backslash E$ is a $\delta$-preclosed set and $D \subset Y \backslash E \subset Y$. This implies that $D$ is not a $\delta$-predense set in $Y$, which is a contradiction. Therefore, $f^{-1}(D)$ is a dense subset of $X$.

$(i i) \Rightarrow(i)$ : Suppose that $D$ is a nonempty open subset of $X$. We want to show that $\delta \operatorname{pint}(f(D)) \neq \emptyset$. Suppose $\delta \operatorname{pint}(f(D))=\emptyset$. Then $\delta p c l(Y \backslash f(D))=$ $Y$. Therefore, by (ii) $f^{-1}(Y \backslash f(D))$ is dense in $X$. But $f^{-1}(Y \backslash f(D)) \subset$ $X \backslash D$. Now $X \backslash D$ is closed. Therefore $f^{-1}(Y \backslash f(D)) \subset X \backslash D$ gives $X=$ $c l\left(f^{-1}((Y \backslash f(D)) \subset X \backslash D\right.$. This implies that $D=\emptyset$ which contradicts the fact that $D \neq \emptyset$. Therefore $\delta \operatorname{pint}(f(D)) \neq \emptyset$. This proves that $f$ is somewhat $\delta$-preopen.

Theorem 3.5. For a bijective function $f:(X, \tau) \rightarrow(Y, \sigma)$, the following statements are equivalent:

(i) $f$ is somewhat $\delta$-preopen;

(ii) If $C$ is a closed subset of $X$ such that $f(C) \neq Y$, then there is a $\delta$-preclosed subset $F$ of $Y$ such that $F \neq Y$ and $f(C) \subset F$.

Proof. $(i) \Rightarrow(i i)$ : Let $C$ be any closed subset of $X$ such that $f(C) \neq Y$. Then $X \backslash C$ is an open set in $X$ and $X \backslash C \neq \emptyset$. Since $f$ is somewhat $\delta$ preopen there exists a $\delta$-preopen set $V$ in $Y$ such that $V \neq \emptyset$ and $V \subset$ $f(X \backslash C)$. Put $F=Y \backslash V$. Clearly $F$ is $\delta$-preclosed in $Y$ and we claim $F \neq Y$. If $F=Y$, then $V=\emptyset$ which is a contradiction. Since $V \subset f(X \backslash C)$, $f(C)=(Y \backslash f(X \backslash C)) \subset Y \backslash V=F$.

$($ ii $) \Rightarrow(i)$ : Let $U$ be any nonempty open subset of $X$. Then $C=X \backslash U$ is closed set in $X$ and $f(X \backslash U)=f(C)=Y \backslash f(U)$ implies $f(C) \neq Y$. 
Therefore, by (ii), there is a $\delta$-preclosed set $F$ of $Y$ such that $F \neq Y$ and $f(C) \subset F$. Clearly $V=Y \backslash F \in \delta P O(Y, \sigma)$ and $V \neq \emptyset$. Also $V=Y \backslash F \subset$ $Y \backslash f(C)=Y \backslash f(X \backslash U)=f(U)$.

Theorem 3.6. Let $f:(X, \tau) \rightarrow(Y, \sigma)$ be somewhat $\delta$-preopen and $A$ be any open subset of $X$. Then the restriction function $f_{A}:\left(X, \tau_{A}\right) \rightarrow(Y, \sigma)$ is somewhat $\delta$-preopen.

Proof. Let $U \in \tau_{A}$ such that $U \neq \emptyset$. Since $U$ is open in $A$ and $A$ is open in $X, U$ is open in $X$ and since by hypothesis $f:(X, \tau) \rightarrow(Y, \sigma)$ is a somewhat $\delta$-preopen function there exists a $\delta$-preopen set $V$ of $Y$ such that $V \subset f(U)$. Thus, for any open set $U$ of $A$ with $U \neq \emptyset$, there exists a $\delta$-preopen set $V$ of $Y$ such that $V \subset f(U)$. This implies that $f_{A}$ is a somewhat $\delta$-preopen function.

Theorem 3.7. Let $f:(X, \tau) \rightarrow(Y, \sigma)$ be a function and $X=A \cup B$, where $A, B \in \tau$. If the restriction functions $f_{A}:\left(X, \tau_{A}\right) \rightarrow(Y, \sigma)$ and $f_{B}:\left(X, \tau_{B}\right) \rightarrow(X, \sigma)$ are somewhat $\delta$-preopen, then $f$ is somewhat $\delta$ preopen.

Proof. Let $U$ be any open subset of $X$ such that $U \neq \emptyset$. Since $X=A \cup B$, either $A \cap U \neq \emptyset$ or $B \cap U \neq \emptyset$ or both $A \cap U \neq \emptyset$ and $B \cap U \neq \emptyset$. Since $U$ is open in $X, U$ is open in both $A$ and $B$.

Case(i): Suppose that $A \cap U \neq \emptyset$ where $A \cap U$ is open in $A$. Since $f_{A}:\left(X, \tau_{A}\right) \rightarrow(Y, \sigma)$ is somewhat $\delta$-preopen, there exists a $\delta$-preopen set $V$ of $Y$ such that $V \subset f(U \cap A) \subset f(U)$, which implies that $f$ is a somewhat $\delta$-preopen function.

Case (ii): Suppose that $B \cap U \neq \emptyset$ where $B \cap U$ is open in $B$. Since $f_{B}:\left(X, \tau_{B}\right) \rightarrow(Y, \sigma)$ is somewhat $\delta$-preopen, there exists a $\delta$-preopen set $V$ of $Y$ such that $V \subset f(U \cap B) \subset f(U)$, which implies that $f$ is a somewhat $\delta$-preopen function.

Case (iii): Suppose that both $A \cap U \neq \emptyset$ and $B \cap U \neq \emptyset$. Then by case (i) and (ii) $f$ is a somewhat $\delta$-preopen function.

Definition 6. A topological space $(X, \tau)$ is said to be $\delta P$-resolvable (resp. resolvable [8]) if there exists a $\delta$-predense (resp. dense) set $A$ in $(X, \tau)$ such that $X \backslash A$ is also $\delta$-predense (resp. dense) in $(X, \tau)$. A space $(X, \tau)$ is called $\delta P$-irresolvable (resp. irresolvable) if it is not $\delta P$-resolvable (resp. resolvable). 
Theorem 3.8. For a topological space $(X, \tau)$, the following statements are equivalent:

(i) $(X, \tau)$ is $\delta P$-resolvable;

(ii) $(X, \tau)$ has a pair of $\delta$-predense sets $A$ and $B$ such that $A \subset(X \backslash B)$.

Proof. $(i) \Rightarrow(i i)$ : Suppose that $(X, \tau)$ is $\delta P$-resolvable. There exists an $\delta$-predense set $A$ in $(X, \tau)$ such that $X \backslash A$ is $\delta$-predense in $(X, \tau)$. Set $B=X \backslash A$, then we have $A=X \backslash B$.

$($ ii $) \Rightarrow(i)$ : Suppose that the statement (ii) holds. Let $(X, \tau)$ be $\delta P$ irresolvable. Then $X \backslash B$ is not $\delta$-predense and $\delta p c l(A) \subset \delta p c l(X \backslash B) \neq X$. Hence $A$ is not $\delta$-predense. This contradicts the assumption.

Theorem 3.9. For a topological space $(X, \tau)$, the following statements are equivalent:

(i) $(X, \tau)$ is $\delta P$-irresolvable (resp. irresolvable);

(ii) For any $\delta$-predense (resp. dense) set $A$ in $X, \delta p i n t(A) \neq \emptyset$ (resp $\operatorname{int}(A) \neq \emptyset)$.

Proof. We prove the first statement since the proof of the second is similar.

$(i) \Rightarrow(i i)$ : Let $A$ be any $\delta$-predense set of $X$. Then we have $\delta p c l(X \backslash A) \neq$ $X$, hence $\delta \operatorname{pint}(A) \neq \emptyset$.

$(i i) \Rightarrow(i)$ : Suppose that $(X, \tau)$ is a $\delta P$-resolvable space. There exists a $\delta$-predense set $A$ in $(X, \tau)$ such that $X \backslash A$ is also $\delta$-predense in $(X, \tau)$. It follows that $\operatorname{spint}(A)=\emptyset$, which is a contradiction; hence $(X, \tau)$ is $\delta P$ irresolvable.

Theorem 3.10. If $f:(X, \tau) \rightarrow(Y, \sigma)$ is a somewhat $\delta$-preopen function and $\delta \operatorname{pint}(B)=\emptyset$ for a nonempty subset $B$ of $Y$, then $\operatorname{int}\left(f^{-1}(B)\right)=\emptyset$.

Proof. Let $B$ be a nonempty set in $Y$ such that $\delta p i n t(B)=\emptyset$. Then $\delta p c l(Y \backslash B)=Y$. Since $f$ is somewhat $\delta$-preopen and $Y \backslash B$ is $\delta$-predense in $Y$, by Theorem $3.2 f^{-1}(Y \backslash B)$ is dense in $X$. Then $c l\left(X \backslash f^{-1}(B)\right)=X$. Hence $\operatorname{int}\left(f^{-1}(B)\right)=\emptyset$.

Theorem 3.11. If $f:(X, \tau) \rightarrow(Y, \sigma)$ be a somewhat $\delta$-preopen function. If $X$ is irresolvable, then $Y$ is $\delta P$-irresolvable.

Proof. Let $B$ be a nonempty set in $Y$ such that $\delta p c l(B)=Y$. We show that $\delta \operatorname{pint}(B) \neq \emptyset$. Suppose not, i.e., $\delta \operatorname{pint}(B)=\emptyset$ then $\delta p \operatorname{pcl}(Y \backslash B)=Y$. Since $f$ is somewhat $\delta$-preopen and $Y \backslash B$ is $\delta$-predense in $Y$, we have by 
Theorem 3.4, $f^{-1}(Y \backslash B)$ is dense in $X$. Then $\operatorname{int}\left(f^{-1}(B)\right)=\emptyset$. Now, since $B$ is $\delta$-predense in $Y$ and using again Theorem $3.4 f^{-1}(B)$ is dense in $X$. Therefore by Theorem 3.9 we have that $\operatorname{int}\left(f^{-1}(B)\right) \neq \emptyset$, which is a contradiction. Hence we must have $\delta \operatorname{pint}(B) \neq \emptyset$ for all $\delta$-predense sets $B$ in $Y$. Hence by Theorem 3.9, $Y$ is $\delta P$-irresolvable.

Definition 7. A function $f:(X, \tau) \rightarrow(Y, \sigma)$ is said to be hardly $\delta$-preopen provided that for each $\delta$-predense subset $A$ of $Y$ that is contained in a proper open set, $f^{-1}(A)$ is $\delta$-predense in $X$.

We have the following characterizations of hardly $\delta$-preopenness.

Theorem 3.12. A function $f:(X, \tau) \rightarrow(Y, \sigma)$ is hardly $\delta$-preopen if and only if $\delta \operatorname{pint}\left(f^{-1}(A)\right)=\emptyset$ for each set $A \subset Y$ having the property that $\delta \operatorname{pint}(A)=\emptyset$ and $A$ containing a nonempty closed set.

Proof. Assume that $f$ is hardly $\delta$-preopen. Let $A \subset Y$ such that $\delta \operatorname{pint}(A)=\emptyset$ and let $F$ be a nonempty closed set contained in $A$. Since $\delta \operatorname{pint}(A)=\emptyset, Y \backslash A$ is $\delta$-predense in $Y$. Observe that $F \subset A, Y \backslash A \subset Y \backslash F \neq$ $Y$. Therefore $f^{-1}(Y \backslash A)$ is $\delta$-predense in $X$. Thus $X=\delta p c l\left(f^{-1}(Y \backslash A)\right)=$ $\delta p c l\left(X \backslash f^{-1}(A)\right)=X \backslash \delta \operatorname{pint}\left(f^{-1}(A)\right)$ which implies that $\delta \operatorname{pint}\left(f^{-1}(A)\right)=$ $\emptyset$.

For the converse implication assume that $\delta \operatorname{pint}\left(f^{-1}(A)\right)=\emptyset$ for every $A \subset Y$ having the property that $\delta \operatorname{pint}(A)=\emptyset$ and $A$ contains a nonempty closed set. Let $A$ be a $\delta$-predense subset of $Y$, that is contained in the proper open set $U$. Then $\delta \operatorname{pint}(Y \backslash A)=\emptyset$ and $\emptyset \neq Y \backslash U \subset Y \backslash A$. Thus $Y \backslash A$ contains a nonempty closed set and hence $\delta \operatorname{pint}\left(f^{-1}(Y \backslash A)\right)=\emptyset$. Then $\emptyset=\delta \operatorname{pint}\left(f^{-1}(Y \backslash A)\right)=\delta \operatorname{pint}\left(X \backslash f^{-1}(A)\right)=X \backslash \delta \operatorname{pcl}\left(f^{-1}(A)\right)$ and hence $f^{-1}(A)$ is $\delta$-predense in $X$.

Theorem 3.13. Let $f:(X, \tau) \rightarrow(Y, \sigma)$ be a function. If $\delta$ pint $(f(A)) \neq \emptyset$ for every $A \subset X$ having the property that $\delta \operatorname{pint}(A) \neq \emptyset$ and there exists a nonempty closed set $F$ for which $f^{-1}(F) \subset A$, then $f$ is hardly $\delta$-preopen.

Proof. Let $B \subset U \subset Y$ where $B$ is $\delta$-predense and $U$ is a proper open set. Let $A=f^{-1}(Y \backslash B)$ and $F=Y \backslash U$. Obviously $f^{-1}(F)=$ $f^{-1}(Y \backslash U) \subset f^{-1}(Y \backslash B)=A$. Also $\delta \operatorname{pint}(f(A))=\delta \operatorname{pint}\left(f\left(f^{-1}(Y \backslash B)\right)\right) \subset$ $\delta \operatorname{pint}(Y \backslash B)=\emptyset$. Therefore we must have that $\emptyset=\delta \operatorname{pint}(A)=\delta \operatorname{pint}\left(f^{-1}(Y \backslash B)\right)=$ $\delta \operatorname{pint}\left(X \backslash f^{-1}(B)\right)$ which implies that $f^{-1}(B)$ is $\delta$-predense. It follows that $f$ is hardly $\delta$-preopen. 
Theorem 3.14. If $f:(X, \tau) \rightarrow(Y, \sigma)$ is hardly $\delta$-preopen, then $\delta$ pint $(f(A)) \neq$ $\emptyset$ for every $A \subset X$ having the property that $\delta$ pint $(A) \neq \emptyset$ and $f(A)$ contains a nonempty closed set.

Proof. Let $A \subset X$ such that $\operatorname{\delta pint}(A) \neq \emptyset$ and $F$ be a nonempty closed set for which $F \subset f(A)$. Suppose $\delta$ pint $(f(A))=\emptyset$. Then $Y \backslash f(A)$ is $\delta$-predense in $Y$ and $Y \backslash f(A) \subset Y \backslash F$ where $Y \backslash F$ is a proper open set. Since $f$ is hardly $\delta$-preopen, $f^{-1}(Y \backslash f(A))$ is $\delta$-predense in $X$. But $f^{-1}(Y \backslash f(A))=X \backslash f^{-1}(f(A))$ and hence $\delta \operatorname{pint}\left(f^{-1}(f(A))\right)=\emptyset$. It follows that $\delta \operatorname{pint}(A)=\emptyset$ which is a contradiction.

Theorems 3.13 and 3.14 are reversible provided that $f$ is surjective. Thus we have the following characterization for surjective hardly $\delta$-preopen functions.

Theorem 3.15. If $f:(X, \tau) \rightarrow(Y, \sigma)$ is surjective, then the following conditions are equivalent:

(i) $f$ is hardly $\delta$-preopen.

(ii) $\operatorname{\delta pint}(f(A)) \neq \emptyset$ for every $A \subset X$ having the property that $\delta$ pint $(A) \neq \emptyset$ and there exists a nonempty closed $F \subset Y$ such that $F \subset f(A)$.

(iii) $\operatorname{\delta pint}(f(A)) \neq \emptyset$ for every $A \subset X$ having the property that $\delta \operatorname{pint}(A) \neq$ $\emptyset$ and there exists a nonempty closed set $F \subset Y$ such that $f^{-1}(F) \subset A$.

Proof. $(i) \Rightarrow(i i)$ : Theorem 3.14

$($ ii $) \Rightarrow($ iii $)$ : Since $f$ is surjective $f^{-1}(F) \subset A$ implies that $F \subset f(A)$.

$($ iii $) \Rightarrow(i)$ : Theorem 3.13.

\section{References}

[1] M.Caldas, T. Fukutake, S. Jafari and T. Noiri, Some applications of $\delta$-preopen sets in topological spaces, Bull. Inst. Math. Acad. Sinica, 33(3), pp. 361-276, (2005).

[2] M.Caldas, T. Fukutake, S. Jafari and T. Noiri, An Alexandroff space defined by $\delta$-preopen sets, Bull. Fukuoka Univ. Ed., 54, Part III, pp. $1-6,(2005)$.

[3] M. Caldas, S. Jafari T.Noiri and M.Simoes, More on contra- $\delta$ precontinuous functions, Miskolc Math. Notes, 9, pp. 25-32, (2008). 
[4] E. Ekici, ( $\delta$-pre,s)-continuous functions, Bull. Malaysian Math. Sci. Soc., 27(2), pp. 237-251, (2004).

[5] E. Ekici, On $\delta$ p-connected spaces, Bull. Carpathian J. Math., in press.

[6] E. Ekici, $\delta$-preopen sets, Mathematica, Tome 47(70), No. 2, pp. 157164, (2005).

[7] K.R. Gently and H.B. Hoyle, Somewhat continuous functions, Czechslovak Math. J., 21, pp. 5-12, (1971).

[8] M. Ganster, Preopen sets and resolvable spaces, Kyungpook Math. J., 27 (2), pp. 135-143, (1987).

[9] N. Levine, Generalized closed sets in topology, Rend. Circ. Mat. Palermo, 19 (2), pp. 89-96, (1970).

[10] S. Raychaudhuri, Concerning $\delta^{*}$-almost continuity and $\delta$-preregularity, Bull. Calcutta Math. Soc., 85, pp. 385-392, (1993).

[11] S. Raychaudhuri and M. N. Mukherjee, On $\delta$-almost continuity and $\delta$ preopen sets, Bull. Inst. Math. Acad. Sinica, 21, pp. 357-366, (1993).

[12] S. Raychaudhuri and M. N. Mukherjee, $\delta_{p}$-closedness for topological spaces, J. Indian Acad. Math., 18, pp. 89-99 (1996).

[13] N. V. Veličko, $H$-closed topological spaces, Mat. Sb., 70 (1966), 98112; English transl., Amer. Math. Soc. Transl., 78, pp. 103-118, (1968).

\section{Caldas}

Departamento de Matemática Aplicada, Universidade Federal Fluminense, Rua Mário Santos Braga, s/n ${ }^{o}$ 24020-140, Niterói,

R.J. Brazil

e-mail: gmamccs@vm.uff.br

and

\section{S. Jafari}

College of Vestsjaelland South, Herrestraede 11, 4200 Slagelse, Denmark

e-mail: jafaripersia@gmail.com 